\title{
L'Afrique naturalisée
}

In: Cahiers d'études africaines. Vol. 39 N¹55-156. 1999. pp. 583-594.

Citer ce document / Cite this document :

Dias Nélia. L'Afrique naturalisée. In: Cahiers d'études africaines. Vol. 39 N¹55-156. 1999. pp. 583-594.

doi : 10.3406/cea.1999.1767

http://www.persee.fr/web/revues/home/prescript/article/cea_0008-0055_1999_num_39_155_1767 


\title{
Résumé
}

Résumé

Il est communément admis que les modes d'exposition des objets dans l'espace des musées ethnographiques nous renseignent tout autant sur les cultures représentées que sur les époques et la culture productrices de ces représentations.

Deux techniques expositives dominent de nos jours la présentation des objets africains dans l'espace des musées : le souci de contextualisation avec une dimension ethnographique (musée de l'Homme) et l'approche esthétique (fondation Dapper, musée des Arts africains et océaniens). Cependant, et au-delà de la dichotomie objet ethnographique/objet d'art, force est de constater que dans certains musées, notamment au musée de la Chasse et de la Nature à Paris, nature et culture semblent aller de pair dans la salle d'Afrique. Ainsi, des lances, des armes, des masques et des sculptures sont exposés à côté de têtes d'animaux, d'animaux empaillés, de cornes d'éléphant en ivoire, de peaux d'animaux, le tout présenté sous forme de trophées de chasse. Si nature et culture sont étroitement liées pour ce qui est de l'Afrique, en revanche la salle d'Asie contient essentiellement des objets de culte. À la fin du XXe siècle les objets africains semblent occuper une position inégale face aux productions matérielles des autres régions géographiques : ce sont quelques-uns des aspects de cette situation que cet article entend examiner.

\begin{abstract}
Abstract

Africa Preserved - The way of displaying objects in folk museums tells us as much about the culture making the exhibit as about the cultures placed on display. Nowadays, museums mainly use two techniques to show African objects: "contextualization" with an ethnological dimension (as in the musée de l'Homme in Paris) and an aesthetic approach (as at the Dapper Foundation and in the musee des Arts africains et oceaniens in Paris). Beyond this art/ethnology dichotomy however, certain museums (for instance, the musée de la Chasse et de la Nature in Paris) seem to fit nature and culture together in the rooms devoted to Africa. Spears, weapons, masks and sculptures are displayed alongside stuffed animals, elephant tusks, hides and skins, ail of which are presented as hunting trophies. In contrast, the Asia room mainly contains religious objects. As the 20th century closes, objects from Asia still seem unequal in position to the material objects from other geographical zones.
\end{abstract}




\section{L'Afrique naturalisée}

Au cœur du Marais, plus précisément au 60 de la rue des Archives, se situe un musée, assez méconnu, qui expose des objets ethnographiques parmi lesquels se tiennent des pièces africaines. Installé dans l'aile gauche de l'Hôtel de Guénégaud, ce musée de la Chasse et de la Nature, inauguré en 1967 par André Malraux, appartient à la fondation de la Maison de la Chasse et de la Nature. On pourrait s'interroger sur les raisons de la présence d'artefacts et d'œuvres d'art dans un musée consacré à la chasse et à la nature. Lors de ma première visite de ce muséc j'ai été frappée par le contraste entre la beauté de l'édifice ( $œ u v r e$ de Mansart), la richesse et la diversité des collections - de la peinture à la tapisserie en passant par la sculpture, les dessins et les armes - et l'étrangeté de la salle d'Afrique, dont le décor exubérant et la contiguité des objets « naturels » et des artefacts n'étaient pas sans rappeler les musées du XIX ${ }^{\mathbb{C}}$ siècle. La profusion d'armes et d'animaux empaillés, le sens du plein, les murs tapissés de lances, de têtes d'animaux et de masques renvoyaient à une sorte de muséographie désuète qui se révélait d'autant plus anachronique étant donné la création de ce musée, à la fin des années 1960. Comment rendre compréhensible, au moment même de la décolonisation, cette représentation de l'Afrique comme un continent "sauvage» et relevant de la "nature"? Quel message était transmis aux visiteurs sur la chasse en dehors de l'Europe et sur les peuples non européens? De plus, la relation entre les deux termes - chasse et nature - n'est pas sans poser des problèmes. En effet, il semblerait que le partage s'effectue entre, d'une part la nature sauvage, brutale, non domestiquée et, conséquemment, des peuples qui vivent dans un "état de nature », d'autre part la noble conquête de l'homme, bien entendu blanc et occidental, sur les forces de la nature, grâce à son ingéniosité et à son adresse, notamment par le biais de la chasse. Chasse et nature loin de s'opposer se complètent dans la mesure où cette dichotomie renvoie à une autre, celle entre culture et nature.

Dans le hall d'entrée trois panneaux renseignent les visiteurs sur l'histoire du musée, ses fondateurs et les objectifs de la Fondation. Le premier rend compte des buts du musée : "Comment la chasse, l'une des toutes premières activités de l'homme, est devenue un moyen de communier avec la nature, de s'y confronter, de s'y mesurer et de mieux la connaître. " La suite du texte explicite le parcours de l'exposition divisé en cinq 
thèmes: mythes et légendes de la chasse, traditions et pratiques de la chasse $X \mathrm{XI}^{\circ}-\mathrm{XVII^{ \circ }}$ siècles, la représentation de l'animal $X V I^{\circ}-X V I^{\circ}$ siècles, traditions et pratiques de la chasse au $X V I^{\circ}{ }^{r}$ siècle, galerie de trophées à l'ouest du donjon. Curieusement, dans le dépliant en vente à l'entrée du musée et daté de cette année, on ne trouve aucune référence à la galerie de trophées en tant que partie constitutive du parcours. C'est dans la section Faune sauvage de ce dépliant que l'on peut lire : «De magnifiques trophées et animaux en cape figurent dans les salles d'Afrique, d'Asie et d'Amérique avec des armes correspondant à ces territoires. Cet ensemble illustre faune exotique et art cynégétique. »

Le deuxième panneau est consacré à la biographie des fondateurs, François Sommer et son épouse Jacqueline, accompagnée d'un grand nombre de photographies du couple. Celle-ci nous apprend qu'ils ont beaucoup voyagé, que Jacqueline Sommer a été à l'origine de l'Association française de la chasse photographique et que Jacques Sommer, grand industriel lié à la manufacture de feutres de Mouzon, fut «l'un des premiers à instituer l'intéressement de ses employés au résultat de l'entreprise ». Amour de la nature et «bien-être » des employés vont de pair, préservation de l'ordre «naturel» et maintien de l'ordre social se conjuguent dans un univers supposé être sans violence. En effet, à aucun moment la mort des animaux n'est mentionnée ${ }^{1}$ et tout se passe comme si protection de la nature et extermination de celle-ci constituaient deux versants d'un même mouvement.

Le troisième panneau fait l'historique de la fondation de la Maison de la Chasse et de la Nature, de ses activités, du parc Bel-Val et des négociations avec la Ville de Paris qui a loué l'Hôtel de Guénégaud à la Fondation par bail emphytéotique à 99 ans. Avant de commencer la visite du musée, les visiteurs sont déjà instruits sur le prestige du bâtiment, la valeur des collections, l'importance de l'ceuvre accomplie par la Fondation - un «club privé de chasseurs et de protecteurs de la nature »²-, le musée de la Chasse et de la Nature et son double, à savoir le parc BelVal qualifié de «musée vivant de la faune sauvage » et de "véritable atelier de réflexion et de formation aux techniques de la chasse de demain, associant tous les acteurs de la nature $2^{3}$. Que la démarche de préservation soit étroitement associée à l'acte de destruction est une évidence sur laquelle, d'ailleurs, s'est forgée la notion de patrimoine. La spécificité de ce musée réside non pas tant dans la prise en compte de ces deux aspects, mais dans le fait que c'est une même institution, en l'occurrence la

1. Susan Sontag (1990): 14-15) a mis en évidence aussi bien les affinités implicites qu'explicites, entre la caméra et lacte de tuer: "The camera is a sublimation of the gun » et "One situation where people are switching from bullets to film is the photographic safari that is replacing the gun safari in East Africa."

2. Musée de la Chassé é de la Nature, brochure. Paris. Nouvelles éditions latines. 1991. p. 7.

3. Musée de la Chassé ét de la Naturé dépliant. 
Fondation de la Chasse et de la Nature, qui prend en charge tout, à la fois des animaux vivants et des animaux empaillés, c'est-à-dire du musée « vivant » et du musée « mort ».

\section{La visite du musée}

Le rez-de-chaussée du musée est consacré, d'après la brochure ${ }^{+}$, au thème de la chasse, du Moyen Âge au XVI' siècle, à l'aide de peintures, sculptures, tapisseries, dessins, armes de chasse, couteaux et tapisseries de Flandres. Cependant, lors de mes visites en août 1998, seules quelques pièces étaient exposées: un tableau de l'École de Flandres représentant la chasse au renard, un portrait de Philippe le Beau par le Maître de la Légende de Madeleine, un bronze de François $1^{\text {er }}$ du XIX ${ }^{\mathrm{e}}$ siècle, un dessin d'un lion couché par Rembrandt. Sur le mur, au fond de la salle, est accroché un tableau de Bernard Lorjou (1908-1986), huile sur toile, représentant un lion, et à côté une lance, posée verticalement, porte la mention «Zäre $\mathrm{XX}$ " siècle. Fer et bois $»$. Au pied de l'escalier on peut voir une défense de narval et sur le mur un extraordinaire bois de cerf gravé représentant des vues de villes du Wurtemberg, datant de 1627. Des études d'animaux par Alexandre-François Desportes sont accrochées tout au long de l'escalier qui mène aux étages supérieurs.

L'itinéraire de l'exposition suit un ordre chronologique qui rend compte de l'histoire, essentiellement technique, de la chasse. A l'entresol est exposée la collection d'armes de chasse et d'armes à feu datant du XVI' au XIX ${ }^{\circ}$ siècle. Outre des armes, on peut y voir, dans des vitrines tapissées, des poires à poudre et des bandoulières de garde-chasse. La muséographie de ces deux salles est sobre, la lumière tamisée et les cartels donnent des informations essentiellement historiques. L'accent est mis sur les instruments de chasse et la cible de la chasse, la bête est physiquement absente mais implicitement présente par des représentations - ainsi les armes et les poires à poudre portent des incrustations à motifs animaliers.

Il en est tout autrement au premier étage; ici et selon la brochure « sans disparaître, les armes vont céder la première place à la faune et à la nature sauvage ${ }^{5}$. À gauche en montant l'escalier se trouvent les salles d'Asie et d'Amérique, et à droite celle d'Afrique, la salle 4, intitulée « La chasse en Afrique » : c'est un long espace de couleur claire dont le plafond a été peint par Bernard Lorjou'. Les couleurs du plafond sont chaudes - des jaunes, rouges et verts - comme pour mieux rappeler la végétation luxuriante de l'Afrique et pour mettre en conformité le décor avec le contenu de la salle. Les motifs du plafond représentent des animaux stylisés

4. Musée de la Chasse et de la Nature, brochure, op. cit., pp. 10-11.

5. Ibid., p. 19.

6. Une exposition consacrée à Bernard Lorjou, Faune sauvage: peintures, est installée, depuis septembre 1998, au musée de la Chasse et de la Nature. 
- des félins? - et des statuettes avec les visages et les corps peints, du type « fétiches ». Les murs sont tapissés de peaux de lions et de têtes d'animaux montés - gazelles, girafes, rhinocéros —, de « massacres » mélangés avec des lances, des masques et des statuettes. Aucun espace n'est laissé libre sur les murs et les visiteurs peuvent se promener tout au long de cette salle rectangulaire. Une estrade longe le mur de droite sur laquelle on peut voir, entre autres, une panthère, un mouflon à manchettes, un lion, des statuettes et une coupe de chef. Aucun ordre classificatoire ne semble régner ici si ce n'est la disposition des artefacts et des animaux pour le plaisir des yeux. Au-dessus de la porte d'entrée sont exposés un masque nalou, un bubale du Tchad, des lances, des arbalètes, des carquois à flèches et des masques sans indication de provenance géographique. Un couple de gorilles du Gabon est présenté au fond de la salle, au milieu d'un feuillage artificiel, contre un mur orné de lances. Au-dessus d'eux une panthère juchée sur un tronc d'arbre semble prête à bondir. Sur le mur de gauche, près des fenêtres, on aperçoit des couteaux de jet et des sagaies, et, sur des socles, deux terres cuites représentant un lion et une panthère de l'École française du XIXe siècle. Animaux empaillés, têtes d'animaux montés, "massacres » confèrent à cette salle une étrange atmosphère, faite d'un mélange d'exotisme et de couleur locale.

Quelques observations s'imposent. Premièrement, il y dans cette salle une totale absence de hiérarchie : les artefacts - lances ou statuettes les peaux d'animaux et les têtes d'animaux montés sont mis sur un même plan. Parfois ce sont les artefacts qui sont difficilement visibles en raison du premier plan occupé par un animal. Aucune pièce africaine ne fait l'objet d'attention particulière, moyennant un dispositif muséographique spécifique : isolement spatial, éclairage, cartel détaillé. Mais tout ceci n'est pas surprenant, car l'image de l'Afrique, transmise par cette salle, est celle d'un continent mystérieux, totalement dominé par la nature au point que même les productions matérielles et artistiques sont naturalisées. Deuxièmement, les cartels sont dépourvus d'informations : pour les animaux, outre leur nom et la région où ils ont été abattus, on indique leur poids et les renseignements sur le donateur — nom de celui-ci et nom de la collection. En ce qui concerne les artefacts, la présence d'indications est purement décorative. Pour ne citer que quelques exemples: «Sagaie pour la chasse à l'hippopotame (Bassin du Congo) », " siège et coupe de chef, tribu fon », «statuette d'antilope femelle (coiffure rituelle Bambara) $»$. Deux pièces se distinguent par des notices plus longues: la première, un «Goli, masque agraire baoulé, Côte-d'Ivoire » dont on apprend qu'il représente « la divinité incarnant l'esprit des morts goli » et qu'il est "utilisé par les confréries agricoles lors des cérémonies agraires ». On peut s'interroger sur la place d'un tel masque, utilisé lors des travaux agricoles, dans une salle consacrée à la chasse. La réponse est simple et se trouve dans le texte même du cartel : " don du Président Senghor. »I a seconde, qui fait l'objet de longues descriptions, est exposée 
dans une vitrine: il s'agit d'un «manteau de chef des chasseurs de la tribu guerzé (Guinée française) ». Le texte donne des informations sur le contexte d'utilisation de ce manteau, sa fonction et les matériaux utilisés. Un tel foisonnement de détails ne gagne de sens que par rapport au donateur du manteau : «... offert en 1949 par les chefs des tribus guerzé à Mr. Roland Pré, gouverneur de la Guinée française de l'époque. C'est un cadeau de reconnaissance pour ce qui avait été fait en faveur de ces populations: maintien de leurs conditions traditionnelles d'existence et protection de leurs zones d'activité de chasse. » Autrement dit, les artefacts ne sont pas importants en eux-mêmes, mais en tant qu'évidences de la prodigalité des donateurs et des réseaux de sociabilité impliqués dans l'activité de la chasse. Troisièmement, les armes «indigènes » comportent rarement des notices, réduites le plus souvent à une simple désignation : « sagaies », «lances », « carquois et flèches». Il en est tout autrement pour les armes à feu dont les notices sont assez longues : « carabine semiautomatique, gros calibre, de l'ancien chasseur professionnel sud-africain tué par un éléphant en 1957 », "fusil ayant servi pour la chasse aux grands animaux », «fusil du chasseur de lions vers $1860 »$, « arme double de gros calibre, utilisée par les Boers d'Afrique du Sud vers $1860 »$. C'est dire que seules les armes utilisées par des Blancs sont susceptibles d'attirer l'attention des visiteurs qui, bien entendu, sont censés partager la culture dont est issu le musée.

Il semble donc que dans cette salle d'Afrique le but n'est pas de rendre compte de la «chasse en Afrique », mais de témoigner de la chasse des Blancs en Afrique. Que ce soient les têtes d'animaux ou les fusils, ce qui est célébré dans cet espace c'est la victoire de la culture blanche sur la nature africaine: les fusils sont des trophées au double sens du terme et commémorent la victoire sur les animaux, en même temps qu'ils célèbrent les propriétaires de ces armes, grâce auxquelles ils ont pu obtenir un tel triomphe, même si, très souvent, ce fut aux dépens de leur vie. L'Afrique représentée dans cette salle est celle des chasseurs, de ceux pour qui ce continent était un terrain vierge, n'attendant qu'à être « découvert » pour consommation. En un certain sens, les chasseurs, qui sont aussi donateurs, participent en tant qu'acteurs au processus de célébration de la chasse et en sont les destinataires. C'est en fin de compte au chasseur, non pas anonyme, mais personnalisé — d'où le rôle des labels précisant qui a tué les animaux - qu'est dédié ce musée. Témoin de l'emprise de l'homme sur le monde naturel, le chasseur est évidemment un homme blanc, aristocrate, explorateur ou gouverneur. Son nom est visible et lisible dans la salle d'Afrique : «célèbre chasseur de lions Jules Gérard » et il n'est pas sans intérêt de noter que dans les deux seules allusions aux Africains, ceux-ci, étant bien entendu anonymes, sont désignés par le terme «indigènes ». Par exemple, dans la brochure du musée on peut lire : «un fusil fabriqué par un indigène qui ne s'en servit qu'une fois, le canon, mal fixé sur le fût, l'ayant tué au premier coup; un autre fusil de fabrication 
indigène, à piston, dont le fût éclaté a été remplacé par un tube de cadre de bicyclette " ${ }^{7}$. En d'autres termes, les références faites aux «indigènes" véhiculent des connotations négatives sur les peuples africains, dont on ne tient compte que des "ratés». De plus, la présence d'artefacts a le statut d'évidence sur les modes de vie des peuples africains, sur leur « nature ». Ainsi les statuettes bambara représentant des antilopes, la coupe

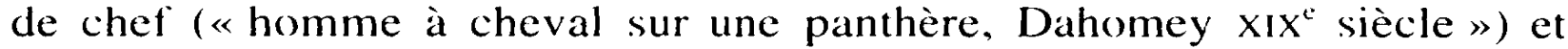
les poids baoulé de mesure ne sont exposés qu'en raison des motifs «animaliers » qui y sont figurés. Pour ce qui est des poids baoulé on peut lire: "Les animaux sont traités avec un sens aigu de l'observation, parfois en stylisant certains détails. " Autrement dit, les objets ne sont pas exposés en raison de leurs qualités formelles et esthétiques, mais en tant que marques du caractère réaliste de la représentation, même si certains détails sont stylisés. Il n'est pas sans intérêt de ne noter que la présence de deux vitrines, la première contenant le manteau du chef guerzé, un masque gouro. les poids baoulé et les poires à poudre; la seconde exposant des sculptures d'animaux, essentiellement des gorilles, par le sculpteur non africain Marcel Lemar. Ces dernières sculptures. d'un réalisme frisant l'exotisme de pacotille, sont bien séparées du reste des objets de la salle, c’est-à-dire de la "nature ", et isolées dans une vitrine.

En sortant de la salle d'Afrique on entre dans une autre, deux fois plus petite, celle de l'Amérique. Un immense ours blanc de l'Alaska entouré d'un ours brun, des têtes de cerf's, des mouflons, des lynx accueillent les visiteurs. Cette salle, aux murs tapissés de bois clair, contient très peu d'objets, exposés surtout dans des vitrines: il s'agit d'antiquités, plus précisément d'objets en terre cuite (un certain nombre d'entre eux étant des vases funéraires), provenant pour la plupart du Mexique. On y trouve également des armes accrochées aux murs et un tableau de George Catlin. représentant des Indiens chassant le bison. Ensuite, nous entrons dans la salle d'Asic aux murs peints de couleur jaune claire. On y voit des têtes montées de mouflon, des cerf's, des tigres. des buffle d'eau, un tigre entier empaillé et des massacres soigneusement arrangés; des labels en gros caractères nous renseignent sur les donateurs des trophées: «Buffle d'eau d'Indochine. Tué par M. de Lesseps », prince d'Iran et des aristocrates. Par terre se trouvent deux tigres en bronze de l'Inde du XVIII" siècle, et accrochée au mur une sarbacane de Malaisie offerte par Charles Ratton. Tous les autres objets sont exposés dans des vitrines. La lecture de la brochure éclaire sur l'esprit qui a présidé à l'installation de cette salle: "Salle d'Asie. Le contraste avec la salle qui précède fil s`agit de la salle d'Amériquel saute aux yeux. Nous entrons dans une civilisation raffinée, dont nous admirons surtout l'art pictural et sculptural ${ }^{x}$. De plus, et à la différence de l'Afrique où la représentation des animaux passe par la

7. Voir hrochure. op. cil., p. 22.

8. Ibid., p. 23. 
stylisation de certains détails (voir pour les poids baoulé), on est ici face à un "souci extrême-oriental de la précision dans le détail »" ${ }^{9}$ Or, cette exactitude dans le rendu des dessins, des gravures, des miniatures et des assiettes requiert une certaine stratégie muséographique : rideaux aux fenêtres, absence de végétation, sobriété dans la décoration de la salle et recours à des vitrines. L'effet de chef-d'œuvre impliquant contemplation et admiration se manifeste dans la présentation des objets en vitrines qui privilégient l'observation minutieuse, spécifiant une certaine classe d'objets comme dignes d'être vus. Comme il s'agit d'une "civilisation raffinée » les objets sont historiquement datés et pourvus de cartels, parfois assez détaillés comme c'est le cas pour le casque à décor de scène de chasse de Perse du XviII ${ }^{\mathrm{e}}$ siècle.

La salle d'Asie communique avec les salles 9 « Les peintres animaliers français », 10 «Salon Desportes» et 11 «Chasse au XIX siècle». Des tableaux, des dessins, des porcelaines et des armes abondent dans ces trois salles où les animaux font défaut, exception faite de deux têtes de cerf datant du XVIII ${ }^{\mathrm{e}}$ siècle. C'est dire que, comme pour les autres salles relatives à l'Europe, la nature n'est donnée à voir que sous sa forme apprivoisée, à savoir la chasse, et la totale absence d'animaux empaillés ou de massacres (sauf les deux têtes de cerf, mais il s'agit de pièces historiques) vient conforter l'emprise de l'art et de la culture sur la nature, ce qui d'ailleurs coïncide avec la fin du parcours de l'exposition.

\section{Nature et culture}

On pourrait, en un certain sens, avancer que ce musée entend essentiellement rendre compte de l'art et de la nature et que la chasse n'y est présente que par le biais de l'art de la chasse. De ce point de vue le dernier paragraphe de la brochure est riche en enseignements : « [...] la visite de ce musée où, d'une part, l'histoire des armes de chasse est si savamment illustrée, où, d'autre part, la nature "sauvage" est si artistiquement présentée ${ }^{10}$. Quatre remarques peuvent être avancées :

- Le recours à des styles muséographiques différents en fonction des aires géographiques est évident. Les techniques de présentation vont de pair avec la hiérarchie des continents; ainsi les vitrines prédominent dans la salle d'Asie et dans celles d'Europe et font défaut dans la salle d'Afrique. La sobriété des salles historiques contraste avec la profusion d'objets dans la salle d'Afrique. L'image de l'Afrique transmise par cette salle est celle d'un continent régi par une vie chaotique où productions matérielles et animaux se confondent dans l'attente d'une mise en ordre venue, bien entendu, de l'Occident. Alors que la muséographie des salles historiques

9. Ibid.

10. Ibid., p. 31. 
vise la contemplation des pièces, en raison de leurs qualités esthétiques et/ou historiques, dans la salle d'Afrique le dessein est d'immerger les visiteurs dans l'exotisme. Et c'est ici que l'art vient au secours de la nature, dans la mesure où la nature « sauvage» est esthétisée. Mais ce processus d'esthétisation ne découle pas des artefacts africains en euxmêmes, mais de leur présentation et agencement dans l'espace du musée pour le plaisir des yeux. En effet, le but de la longue salle d'Afrique (avec une seule porte servant à la fois d'entrée et de sortie) est de transporter les visiteurs, comme au XIX ${ }^{\circ}$ siècle, dans un autre espace géographique. De plus, ce voyage dans l'espace est doublé d'un voyage touristique, du genre safari. Or, ce qui rend possible cette immersion dans l'exotisme c'est le dispositif d'exposition moyennant l'utilisation abondante des trophées d'animaux.

- La vie des animaux a été appropriée par la taxidermie à des fins d'exposition. Grâce à celle-ci, les bêtes, quoique mortes, semblent immortelles, en raison de leurs yeux en émail, de leurs poses mouvantes et de leurs bouches ouvertes. Donna Haraway (1984/85) a remarquablement montré que la taxidermie est par excellence au service du réalisme, fonctionnant un peu comme un peephole into reality. C'est comme si ces animaux étaient là, juste pour être regardés, dans leur état de "nature ». Or, ce que l'on voit ce ne sont pas les animaux, mais le produit des opérations de mise à mort et de dépeçage et le travail de taxidermie pour la mise en exposition: le résultat de tout un processus muséologique qui transforme les animaux en objets culturels. S'il est presque banal de dire que ce qui est donné à voir ce n’est pas la «nature ", mais une certaine représentation de la nature, il n'en reste pas moins que cette salle tente de nous faire croire que ce que l'on voit reproduit fidèlement la nature, que l'efficacité de ce musée passe par son impact visuel. Or, l'un des éléments-clé de la stratégie de l'exposition ce sont les trophées. Bien avant son utilisation dans l'espace muséal. en tant que dispositif, le trophée est une marque commémorative d'une victoire sur les hommes - trophée d'armes - et sur les animaux - trophée de chasse - présupposant donc un acte de violence de la part d'un individu de sexe masculin et l'appropriation d'un butin. Comme l'a montré John Mackenzie (1988: 28-31) l’introduction d'animaux empaillés. sous forme de trophées. dans la décoration d’intérieurs, a été faite graduellement et, quoique difficile à dater. elle est en grande partie le produit de l'imagination romantique qui voit son aboutissement à l’Exposition universelle de 1851 au Crystal Palace. Selon lui, culte de la chasse et pouvoir impérial vont de pair au XIX" siécle et s’expriment l'un et l’autre dans et par les trophées. Rien d’étonnant alors qu'au XIX" siècle les musées militaires et ethnographiques aient adopté le trophée comme technique dexposition. Mais é qui rend plus problématique la présence de trophées au musée de la Chasse et de la Nature ceest leur abondance dans les salles d'Alrique. d'Amérique et d'Asie el leur relative absence dans les salles d'Europe (exception faite 
des têtes de cerf exposées en raison de leur qualité esthétique - bois gravé - et de leur importance historique). Si en Europe la chasse est historicisée (voir le parcours allant du XIV" au XIX ${ }^{\circ}$ siècle). elle est par contre naturalisée pour ce qui est des autres régions géographiques.

- À la dichotomic chasse/nature, sen superpose une autre qui concerne les rapports entre histoire et nature. Les techniques de chasse actucllement en cours en Europe 11 ne sont pas représentées dans l'espace du musée. comme si cette pratique s'était arrêtée au XIX ${ }^{\circ}$ siècle : cependant il n'en reste pas moins qu'à la mise en contexte historique de la chasse en Europe s'oppose la totale absence de dimension historique en ce qui concerne la salle d'Afrique. On ne sait presque rien sur la période à laquelle appartiennent les armes africaines et d'ailleurs ce type de renseignement cst, dans la logique du musée, presque superflu, étant donné que les objets appartiennent à des peuples de la nature, c'est-à-dire hors de l'histoire. Autrement dit, la culture occidentale existe dans l'histoire et possède une dynamique, à la différence de la nature (et des peuples de la nature) qui est statique et atemporelle ${ }^{12}$.

- La dernière remarque concerne la notion de chasse. D’une façon générale ce musée contient d'une part, les instruments, les armes qui servent à chasser, de l'autre, le résultat de la chasse, les bêtes mortes sous forme de trophées. Tout ce qui s'accomplit entre ces deux phases, comment on chasse, quand on chasse, quels groupes sociaux et sexuels sont impliqués, quel type de gibier, quelles périodes de l'année, les manifestations sociales et symboliques qui y sont associées, est totalement passé sous silence. Peut-on parler de la chasse comme d'une catégorie générale, indépendamment non seulement des contextes géographiques mais aussi des cadres sociaux? Peut-on mettre sur un même plan et au sein de la catégorie chasse des activités aussi diverses que la capture des animaux pour des raisons alimentaires et la poursuite des animaux en tant qu'activité sportive? Qu'y a t-il de commun entre la chasse collective et celle pratiquée par petits groupes? Peut-on désigner comme «armes indigènes » aussi bien ce qui relève de la chasse avec des armes, les « harpons pour chasse à l'hippopotame », que ce qui est de l'ordre de la chasse avec des pièges, le «piège de bois avec montage en lanières de boyau »"13 ? Comme l'avait déjà noté Marcel Mauss (1967 : 58-60), il est nécessaire de distinguer la chasse active de la chasse passive, la première impliquant la présence et

11. Pour une approche de la chasse en France voir l'ouvrage de Bertrand HELL (1985) qui contient, entre autres, des pages instructives sur le culte du trophée, en particulier les trophées de cerf, et le symbolisme sous-jacent associé aux thèmes de la mort, de la virilité et de la sexualité.

12. Cette tension entre nature et culture a été remarquablement analysée par Mieke BAL (1996: 13-56) pour les collections africaines de l'American Museum of Natural History à New York.

13. Voir brochure, op. cit., p. 22. 
la participation active de l'homme (isolé ou en groupe), alors que dans la seconde, identifiée dans la chasse avec des pièges, l'homme est passif ${ }^{14}$.

Comment rendre intelligible la présence de trophées dans les salles de ce musée ? S'il est vrai que, d'un certain point de vue, la salle d'Afrique partage certaines conventions de représentation propres aux modes d'exposition du $\mathrm{XIX}^{\mathrm{E}}$ siècle, on ne peut cependant pas parler d'anachronisme. Conçu en 1967, c'est-à-dire au moment de la décolonisation, ce musée est, par son mode d'exposition, le choix des objets, le type d'information transmise et les termes utilisés pour caractériser les objets, un musée qu'on pourrait qualifier de colonial, dans la mesure où il partage des conventions de présentation et de représentation propres à la culture du colonialisme. Ceci ne veut pas dire pour autant qu'un esprit colonialiste ait présidé à la fondation de ce musée et guidé ses modes de présentation; dans ces conditions, pourquoi, alors que le colonialisme a disparu en tant que système politique, les catégories du colonialisme persistent et continuent encore à façonner les modes de présentation des objets, leur choix tout comme les termes employés pour désigner les artefacts, et ce même dans le cadre des musées ethnographiques ${ }^{15}$. Ne faudrait-il pas chercher l'une des explications possibles du côté de la persistance dans la culture visuelle ${ }^{16}$ d'un certain nombre de stéréotypes qui, consciemment ou inconsciemment, reproduisent et renforcent les inégalités en termes de différences ethniques?

Lors de l'une de mes visites au musée, j'ai fait la connaissance du taxidermiste, Jacques Barboni, qui, avec beaucoup de gentillesse, m'a fourni de très utiles renseignements. Il a réalisé la grande majorité des travaux de taxidermie du musée; interrogé sur les critères présidant au choix de l'emplacement des pièces dans la salle d'Afrique, il a répondu très explicitement que c'est en travaillant de concert avec Monsieur Sommer, qu'ils ont disposé les animaux et les objets en fonction d'abord de leur taille (les grandes et les plus importantes pièces, comme la tête de rhinocéros noir du Kenya, au premier plan, les lances tout en haut des murs, les sagaies dans les espaces laissés libres) puis de leur impact visuel. C'est dire que des facteurs tels que les expériences visuelle et technique antérieures, l'éducation de l'œil, la pratique de safaris et la fréquentation de zoo, ont présidé, tout naturellement, à ce type de présentation. Vouloir attribuer un dessein idéologique délibéré, c'est en fin de compte sous-estimer les relations étroites qui unissent le musée et le «monde

14. Pour un traitement plus systématique des différences entre la chasse et le piégeage, voir Jean JAMIN (1979: 22-29).

15. Sur cette question, et pour une approche générale, voir l’importante introduction de Frederick Coopl:k et Ann L. STolli:r (1997). Pour ce qui ést des musées ethnographiques, voir Nélia Dias (1998).

16. Entendue au sens large du terme, comprenant les expositions. mais aussi le cinéma. les affiches, les illustrations. la publicité el les magazines du type National (jeographic. 
extérieur », relations qui s'expriment non seulement par la façon dont l'effet musée se manifeste dans la sphère du tourisme, des activités ludiques et du commerce - les espaces d'exposition dans les shopping centers ne sont qu'un exemple —, mais aussi par les conséquences qu'induisent un certain nombre de pratiques quotidiennes (visites des jardins zoologiques, lecture de magazines illustrés, projections de films et de séries documentaires sur la nature, etc.) sur les musées. S’il est indéniable que le processus de muséalisation des différentes sphères de la vie quotidienne s'accrô̂t chaque jour, la façon dont la «culture », entendue au sens large du terme, modèle le monde des musées est tout aussi incontestable.

ISCTE, Université de Lisbonne, département d'anthropologie.

\section{B I B LIOGR A P H I E}

BAI, $M$.

1996 «Telling, Showing, Showing Off», in M. BAL, Double Exposures : The Subject of Cultural Analysis, New York, Routledge : 13-56.

COOPIR, F. \& STOL.FR, A. L., eds

1997 «Between Metropole and Colony. Rethinking a Research Agenda», in F. COOPER \& A. L. STOLER, eds, Tensions of Empire: Colonial Cultures in a Bourgeois World, Berkeley, University of California Press : 1-56.

DIAS, N.

1998 «Musée ethnographique, musée colonial », in Du Musée colonial à..., Colloque, 3-6 juin, Paris, Centre Georges Pompidou/musée des Arts africains et océaniens.

HARAWAY, D.

1984/85 «Teddy Bear Patriarchy Taxidermy in the Garden of Eden New York City, 1908-1936», Social Text, $11:$ 19-64.

HeLL, B.

1985 Entre chien et loup. Faits et dits de la chasse dans la France de l'Est, Paris, Éditions de la Maison des sciences de l'homme.

JAMIN, J.

1979 La Tenderie aux grives chez les Ardennais du plateau, Paris, Institut d'ethnologie.

MACKenZIE, J.

1988 The Empire of Nature: Hunting, Conservation and British Imperialism, Manchester, Manchester University Press. 
MAlss, M.

1967 Manuel d'ethnographie, Paris, Payot.

Sontac; S.

1990 On Photography. New York, Anchor Books (1" éd. 1977).

\section{RÉSUMÉ}

Il est communément admis que les modes d'exposition des objets dans l'espace des musées ethnographiques nous renseignent tout autant sur les cultures représentées que sur les époques et la culture productrices de ces représentations.

Deux techniques expositives dominent de nos jours la présentation des objets africains dans l'espace des musées : le souci de contextualisation avec une dimension ethnographique (musée de l'Homme) et l'approche esthétique (fondation Dapper, musée des Arts africains et océaniens). Cependant, et au-dela de la dichotomie objet ethnographique/objet d'art, force est de constater que dans certains musćes, notamment au musée de la Chasse et de la Nature à Paris, nature et culture semblent aller de pair dans la salle d'Afrique. Ainsi, des lances, des armes, des masques et des sculptures sont exposés à côté de têtes d'animaux, d'animaux empaillés, de cornes d'éléphant en ivoire, de peaux d'animaux, le tout présenté sous forme de trophées de chasse. Si nature el culture sont étroitement liées pour ce qui est de l'Afrique, en revanche la salle d'Asie contient essentiellement des objets de culte. A la fin du $x x^{\prime \prime}$ siecle les objets africains semblent occuper une position inégale face aux productions matérielles des autres régions géographiques: ce sont quelques-uns des aspects de cette situation que cet article entend examiner.

\section{ABSTRACT}

Atrica Preserved. - The way of displaying objects in folk museums tells us as much about the culture making the exhibit as about the cultures placed on display. Nowadays, museums mainly use two techniques to show African objects: "contextualization" with an ethnological dimension (as in the musée de l'Homme in Paris) and an aesthetic approach (as at the Dapper Foundation and in the musee des Arts africains et océaniens in Paris). Beyond this art/ethnology dichotomy however, certain museums (for instance, the muse de la Chasse of de la Nature in Paris) seem to fit nature and culture together in the rooms devoted to Airica. Spears, weapons, masks and sculptures are displayed alongside stuffed animals, elephant lusks, hides and skins, all of which are presented as hunting trophies. In contrast, the Asia room mainly contains religious objects. As the $20^{\text {th }}$ century closes, objects from Asia still seem unequal in position to the material objects from other geographical eones.

Mots-cles/Keywords: chasse, musées ethnographiques, nature/culture, objets africains/humting, musedums, ethmology, nature/culture, objecets. 